\title{
On-orbit Science Unit Maintainance in Space Telescope
}

\author{
Yijian Wang ${ }^{1, a}$, Weiguo Zhao ${ }^{1, b}$, Yanchun $\mathrm{Li}^{1, \mathrm{c}}$, Funan $\mathrm{Yu}^{1, \mathrm{a}}$, Yan Wang ${ }^{1, \mathrm{~d}}$, \\ ${ }^{1}$ Changchun Institute of Optics Mechanics and Physics of Chinese Academy of Sciences, \\ Changchun 130033,China \\ a'wangyijian121@126.com, 'b_w_g@163.com, ${ }^{\mathrm{c}}$ feilong99031@126.com, 'wangyan@ciomp.ac.cn
}

Keywords: on-orbit, science unit, space telescope, maintenance

\begin{abstract}
With the development of space technology, the large diameter long-life of the space telescopes became more and more urgent. This paper mainly discussed the feasibility of the space telescope on-orbit maintenance for science unit module. This paper took the WFC3 module of the Hubble space telescope for example. In this paper, the Hubble space telescope was analyzed for on-orbit maintenance. By discuss the installation of the science unit maintenance, the paper way to achieve space telescope in orbit maintenance.
\end{abstract}

\section{Introduction}

With the development of aerospace technology, it has begun to need more techniques of the space telescope. In recent years, with the objective to enhance the demand for space observation of the space telescope, the pupil diameter and other indicators of the increasingly high demand for the space telescope investment is also improved. If there are some simple problems caused the telescope broken, that will result in large economic losses. That means when some non-fatal failure happens, that the maintain of the space telescope can be done to make the telescope well. On the other hand, with the development of the space telescope and technology, the science unit replacement is inevitable. The telescope upgrade or expand on-orbit can invest less in the case to get more scientific and technological achievements. So the science unit maintains on-orbit can change a lot of the space telescope for our country.

\section{The Kinematic Installation Analysis}

The space telescope science unit model according to optical requirements, needs to have a high positioning accuracy. In the space of freedom limiting module process, if there is too positioning will produce excess stress inside the module is installed, the module installation affect accuracy. During installation can be sufficiently limiting module space six degrees of freedom and does not produce excessive installation called kinematic maintains.

Kinematic installation includes two forms: (1)2-2-2 installation; (2)3-2-1 installation.

\section{1 "2-2-2" installation}

"2-2-2" installation shown in fig 1. This positioning through three A shape legs. One A shape leg confined the module two degrees of freedom, so that the module has a high positioning accuracy and dimensional stability, can be used in sensitive to temperature changes cryogenic systems.

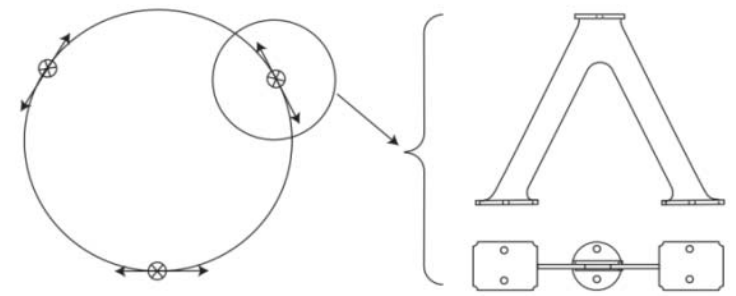

Figure 1 "2-2-2" installation positioning principle 
"2-2-2" installation in three tangentially mounted A shape leg supporting is able to release the stress of the flexible design, installation can be avoided in order to eliminate unnecessary stress increases the mechanical design.

"2-2-2" installation due to the required mounting points are more often used to install and do not require replacement of the rail maintenance module, to avoid increasing the astronauts in orbit operations and the maintenance time. As shown in Figure 2, the ISIM of JWST system, "2-2-2" positioning its use can guarantee the module will not change position due to thermal stress, ensure the module positioning accuracy in space.

\section{2 "3-2-1" installation}

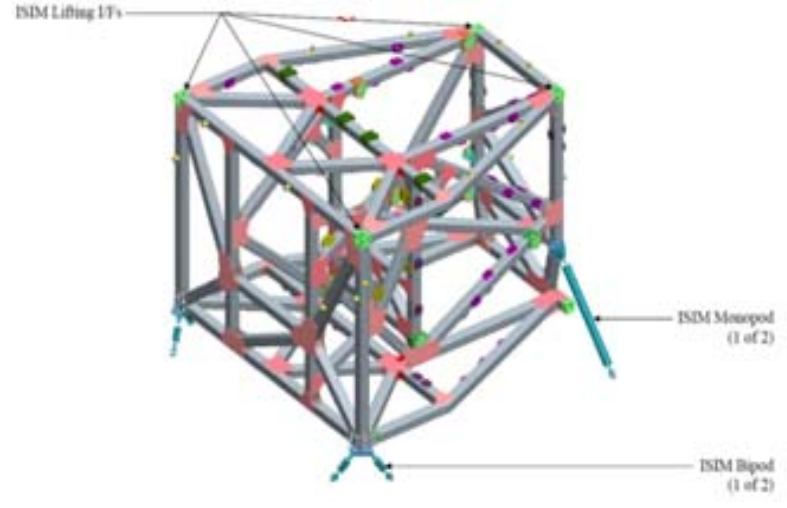

Figure 2 ISIM installation

3-2-1 kinematic installation principle is shown in Fig3. Kinematic positioning method by three points in different ways to restrict freedom of space, rear combination to limit the space six degrees of freedom.

A, B, C three fixed points on the module, A-point through ball joints or short taper constraint module X, Y, Z-axis of the translational degrees of freedom; B points along the Z-axis and Y-axis freedom of being short constraint $\mathrm{V}$ groove, $\mathrm{B}$ point a point interaction module can rotate around the $\mathrm{Y}$-axis and $\mathrm{Z}$-axis degree of freedom of constraints; $\mathrm{C}$ point is located on a plane parallel with the XY plane along the $\mathrm{Z}$-axis translation is constrained, and the point $\mathrm{C}$ and $\mathrm{A}$ point, $\mathrm{B}$ point cooperate together to block freedom of rotation about the $\mathrm{X}$ axis constraints. This joint action by the "point line side" freedom of space limitations method called "3-2-1" positioning method.

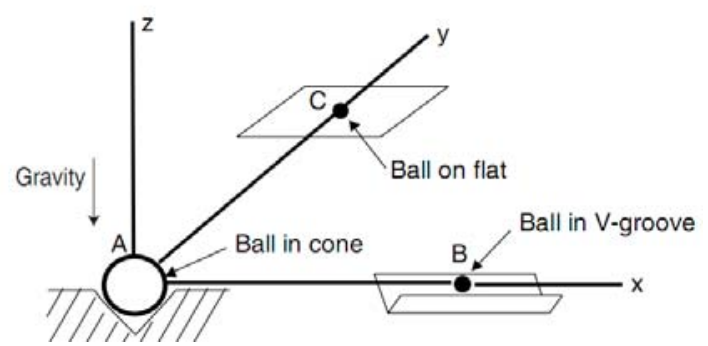

Figure 3 "3-2-1" installation

Only A point positioning in 3-2-1installation requires the pressing force of compression, so in the case of mounting strength can guarantee requires only one installation point can meet the installation requirements. Some can be used as needed in rail maintenance module disassembly and installation of a positioning mechanism, both to ensure the installation accuracy, but also can reduce the astronauts to operate, reduce maintenance difficulty.

\section{WFC3 Module Installation Location}

An example of this arrangement is the WFC3 of the Hubble space telescope. Hubble WFC3 payload replacement mechanism design uses a "3-2-1" positioning kinematics installation, combined with the guide rails module replacement. Replace agency by guiding precision from low to high and have the installation sequence by rough guide rail, B, C pin, fine guide A pin three parts, provided rough guide rail, $\mathrm{B}, \mathrm{C}$ pin, pin locations, such as shown in Fig 4. 


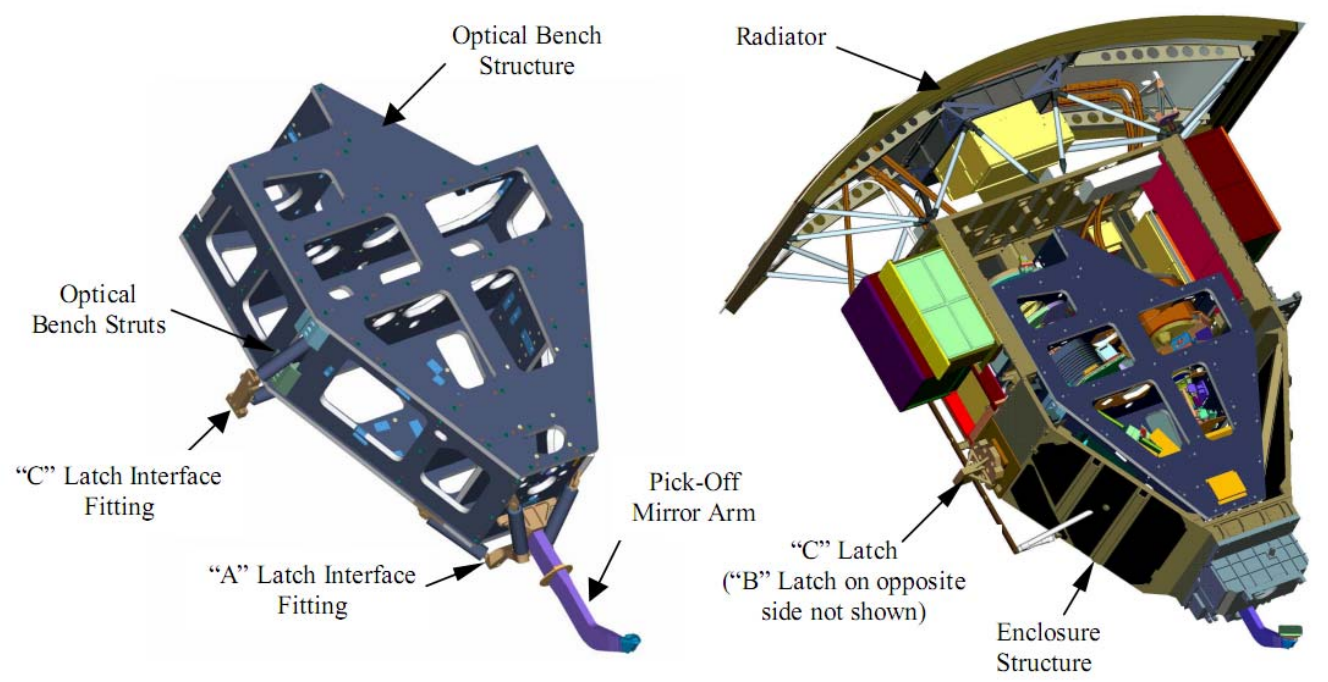

Figure 4 WFC3 Instrument Solid Model View

There are 3 units in WFC3, such as the guide, B\&C point and A point.

a) Guide. To achieve the first stage of the guide, the slider and the rail gap sufficient low guiding precision, easy to find replacement staff, aligned, replace the module.

b) $\mathrm{B} \& \mathrm{C}$ point. Achieve a more level fine positioning, B pin two translational degrees of freedom is restricted, $\mathrm{C}$ pin a translational degrees of freedom is limited.

c) A point. A short tapered pin point positioning, limiting the translational degrees of freedom in three directions, is the key point of the entire module positioning the highest accuracy. The structure shown in Figure 5, the taper pins and springs facility on the cone module and fastening bolts and other components. Positioning, tapered bore and studs rough alignment to be consisting of $\mathrm{B}, \mathrm{C}$ pin positioning accuracy guarantee, after coarse alignment, use the taper pin and the taper hole of self-centering function, to achieve precise positioning taper pin and the contact tapered bore after positioning, tighten the fixing bolts to achieve compression module. Spring when removing modules provide outward force of the auxiliary module smoothly disengaged.

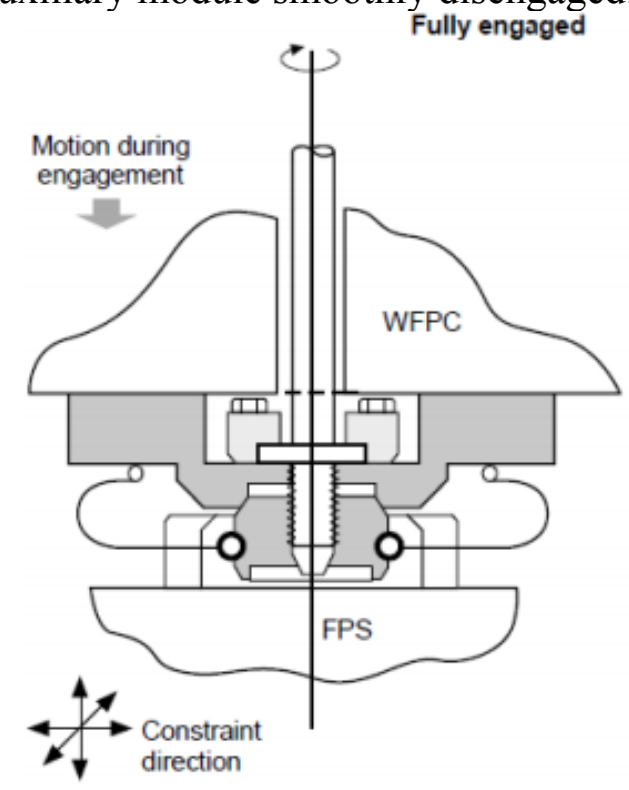

Figure 5 A Point Anomaly

WFC3 modules during the installation process can be seen as a rigid body, as shown in figure $6, \mathrm{~A}$ point positioning accuracy as a module locking highest point in Figure 4, three-axis translational degrees of freedom locked module, $\mathrm{B}$ and $\mathrm{C}$ points during the installation process because installation accuracy limitations, the module can be considered as a rigid body rotation around point $\mathrm{A}$, with the span between the B / C locking point a point scale, improve WFC3 module rotation accuracy. 


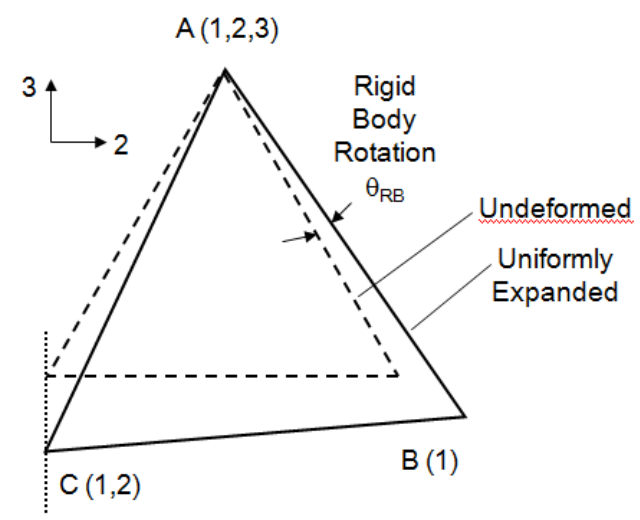

Figure 6 WFC3 module rigid displacement

In the actual design process, A point on the module on a cone and facilities positioning accuracy of Cone pins required by the manufacturing process and alignment to be guaranteed, tapered bore relative module image plane, taper pin relative to the optical system of the position and attitude are required in the alignment process make adjustments, and the need to image the result as one of the means of detection accuracy of the positioning module.

\section{CONCLUSIONS}

Space Telescope's large-scale, long service cycle design space telescope has increasingly become an important part of the process, but due to different life space telescope constituent units, and therefore their life restricted by the smallest unit of life. Through the implementation of on-orbit maintenance of the space telescope, the space telescope to maximize the promotion of life, to ensure its rail service time.

Through the analysis of the Space Telescope in orbit positioning installation, it discusses the basic ideas of space telescope backend module installation location. Meanwhile, the Hubble Space Telescope WFC3 positioning module is analyzed for future space telescope in orbit maintenance of the design basis.

\section{References}

[1].Daniel Musinski, Hemy Sampler, John Evans, Metrology for the Flight Instruments for the Hubble Space Telescope First Servicing Mission at NASA/Goddard Space Flight Center. SPIE(2012) 167-174.

[2].Cengiz Kunt, Swales Aerospace, Beltsville, WFC3 OPTICAL BENCH STRUCTURAL THERMAL OPTICAL PERFORMANCE (STOP) ANALYSIS AND OPTIMIZATION, AIAA 2003-1530

[3].Kirk K. Rhee, Mark A. Neuman, Peter M. Alea, Hubble Space Telescope Extravehicular Activity Tools and Crew Aids Design., AIAA 94-0088

[4].Jill Holz et al., WFC3 Optical Bench, Critical Design Review, NASA GSFC, June 2000. 